\title{
Asymptotic Stability and Decay Rates of Positive Linear Systems with Unbounded Delays
}

\author{
Hamid Reza Feyzmahdavian, Themistoklis Charalambous and Mikael Johansson
}

\begin{abstract}
There are several results on the stability analysis of positive linear systems in the presence of constant or timevarying delays. However, most existing results assume that the delays are bounded. This paper studies the stability of discrete-time positive linear systems with unbounded delays. We provide a set of easily verifiable necessary and sufficient conditions for delay-independent stability of positive linear systems subject to a general class of heterogeneous timevarying delays. For two particular classes of unbounded delays, explicit expressions that bound the decay rate of the system are presented. We demonstrate that the best bound on the decay rate that our results can guarantee can be found via convex optimization. Finally, the validity of the results is demonstrated via a numerical example.
\end{abstract}

\section{INTRODUCTION}

Positive systems are dynamical systems whose state variables are constrained to be nonnegative for all time whenever the initial conditions are nonnegative [1]. Due to their importance and wide applicability, the analysis and control of positive systems has attracted considerable attention from the control community (see, e.g., [2]-[9] and references therein).

Since time delays are omnipresent in engineering systems, the study of stability and control of dynamical systems with delayed states is essential and of practical importance. For general systems, the existence of time delays may impair performance, induce oscillations and even instability [10]. In contrast, positive linear systems are known to be insensitive to several classes of time-delays [11]-[13] in the sense that a positive linear system with delays is asymptotically stable if and only if the corresponding delay-free system is asymptotically stable.

At this point, it is worth noting that the results for positive linear systems cited above consider bounded delays. However, in some cases, it is not possible to a priori guarantee that the delays will be bounded, but the state evolution might be affected by the entire history of states. It is then natural to ask if the insensitivity properties of positive linear systems with respect to time delays will hold also for unbounded delays. In [14], it was shown that, for a particular class of unbounded delays, this is indeed the case. However, the question remains open for general classes of unbounded delays. Moreover, the impact of unbounded delays on the decay rate of positive linear systems was not considered in [14]. This paper closes these gaps.

H. R. Feyzmahdavian, T. Charalambous, and M. Johansson are with ACCESS Linnaeus Center, School of Electrical Engineering, KTH-Royal Institute of Technology, SE-100 44 Stockholm, Sweden. Emails: \{hamidrez, themisc, mikaelj\}@kth.se.
At the core of our paper is a set of powerful conditions for delay-independent stability of discrete-time positive linear systems with heterogeneous unbounded time-varying delays. Our proof technique, which neither uses the LyapunovKrasovskii functional method used in [11] nor the approach used in [14], allows us to impose minimal restrictions on delays. More specifically, we make the following contributions. First, we derive a necessary and sufficient condition for asymptotic stability of discrete-time positive linear systems with heterogeneous unbounded time-varying delays. Then, for two particular classes of unbounded delays, we present explicit expressions that bound the decay rate of the system. Finally, we demonstrate that the best decay rate that our bounds can guarantee can be found via convex optimization.

The remainder of the paper is organized as follows. Section II gives a description of the problem under consideration, and the main results of this work are presented in Section III. An Illustrative example is presented in Section IV, justifying the validity and benefits of our results. Finally, concluding remarks are given in Section V.

\section{A. Notation}

Vectors are written in bold lower case letters and matrices in capital letters. We have $\mathbb{R}, \mathbb{N}$, and $\mathbb{N}_{0}$ for the set of real numbers, natural numbers, and the set of natural numbers including zero, respectively. The largest integer less than or equal to real number $x$ is indicated by $\lfloor x\rfloor$. The non-negative orthant of the $n$-dimensional real space $\mathbb{R}^{n}$ is represented by $\mathbb{R}_{+}^{n}$. The $i^{t h}$ component of a vector $\boldsymbol{x} \in \mathbb{R}^{n}$ is denoted by $x_{i}$, and the notation $\boldsymbol{x}>\mathbf{0}$ implies that $x_{i}>0$ for all components $i$. Given a vector $\boldsymbol{v}>\mathbf{0}$, the weighted $l_{\infty}$ norm is defined by

$$
\|\boldsymbol{x}\|_{\infty}^{\boldsymbol{v}}=\max _{1 \leq i \leq n} \frac{\left|x_{i}\right|}{v_{i}} .
$$

For a matrix $A \in \mathbb{R}^{n \times n}, a_{i j}$ denotes the entry in row $i$ and column $j$. A matrix $A \in \mathbb{R}^{n \times n}$ is said to be non-negative if $a_{i j} \geq 0$ for all $i, j$. The spectral radius of a matrix $A$, denoted $\rho(A)$, is the largest magnitude of its eigenvalues.

\section{Problem Statement}

In this paper, we consider a discrete-time linear system with heterogeneous time-varying delays given by

$$
\begin{array}{rlrl}
x_{i}(k+1) & =\sum_{j=1}^{n} a_{i j} x_{j}(k)+\sum_{j=1}^{n} b_{i j} x_{j}\left(k-d_{j}^{i}(k)\right), k \in \mathbb{N}_{0}, \\
x_{i}(k) & =\varphi_{i}(k), & k \in\left\{-d_{\max }, \ldots,-1,0\right\} .
\end{array}
$$


Here, $\boldsymbol{x}(k)=\left[x_{1}(k), \ldots, x_{n}(k)\right]^{T} \in \mathbb{R}^{n}$ is the state variable, $A=\left[a_{i j}\right] \in \mathbb{R}^{n \times n}, B=\left[b_{i j}\right] \in \mathbb{R}^{n \times n}$ are system matrices, $d_{j}^{i}(k) \in \mathbb{N}_{0}$ for $i, j=1, \ldots, n$ represent the time-varying delays, $d_{\max } \in \mathbb{N}_{0}$, and $\varphi(\cdot)=\left[\varphi_{1}(\cdot), \ldots, \varphi_{n}(\cdot)\right]^{T}$ is the vector sequence specifying the initial state of the system.

System (1) is said to be positive if for every non-negative initial condition $\varphi(\cdot) \in \mathbb{R}_{+}^{n}$, the corresponding state trajectory is non-negative, that is $\boldsymbol{x}(k) \geq \mathbf{0}$ for all $k \in \mathbb{N}$. Positivity of (1) is readily verified using the following result.

Proposition 1 ( [13]) The discrete-time system (1) is positive if and only if $A$ and $B$ are non-negative.

It should be stressed here that the non-negativity of the initial condition is essential for ensuring positivity of the state evolution of (1). In other words, when $\varphi(\cdot) \geq 0$ is not satisfied, $\boldsymbol{x}(k)$ may not stay in the positive orthant even if the conditions of Proposition 1 hold.

While $\boldsymbol{x}=\mathbf{0}$ is clearly an equilibrium point of the system (1), it is not necessarily stable. Moreover, the stability of general linear systems may depend on the magnitude of the time delays. However, as discussed in the introduction, the asymptotic stability of discrete-time positive linear systems with bounded delays $\left(d_{j}^{i}(k) \leq d_{\max }\right)$ does not depend on the magnitude of the delays, only on the system matrices [11][13]. It is then of great interest for theory and practice to determine if a similar delay-independent stability result holds for discrete-time positive systems with unbounded delays.

A general class of time delays is described by the following assumption.

Assumption 1 The delays $d_{j}^{i}(k)$ for $i, j=1, \ldots, n$ satisfy

$$
\lim _{k \rightarrow+\infty} k-d_{j}^{i}(k)=+\infty
$$

Roughly speaking, Assumption 1 says that as $k$ increases, the delays $d_{j}^{i}(k)$ do not grow at the same rate as $k$. It is easy to see that all bounded delays, irrespective of whether they are constant or time-varying, satisfy this assumption. Note also that delays satisfying (2) may be unbounded. A particular class of unbounded delays was considered in [14] and is specified by Assumption 2 .

Assumption 2 There exist $T \in \mathbb{N}$ and a scalar $0<\alpha<1$ such that the delays $d_{j}^{i}(k)$ satisfy

$$
\sup _{k>T, i, j} \frac{d_{j}^{i}(k)}{k}=\alpha .
$$

One can easily verify that constraint (3) on delays implies (2). However, the following example shows that the converse does not hold in general.

Example 1 Let $d_{j}^{i}(k)=k-\lfloor\sqrt{k}\rfloor$ for $k \in \mathbb{N}_{0}$. Since

$$
\begin{aligned}
\lim _{k \rightarrow+\infty} k-d_{j}^{i}(k) & =\lim _{k \rightarrow+\infty}\lfloor\sqrt{k}\rfloor=+\infty, \\
\lim _{k \rightarrow+\infty} \frac{d_{j}^{i}(k)}{k} & =\lim _{k \rightarrow+\infty} \frac{k-\lfloor\sqrt{k}\rfloor}{k}=1,
\end{aligned}
$$

it is clear that (2) holds while (3) does not hold.

To the best of our knowledge, Assumption 1 is the weakest restriction for delays that can still guarantee asymptotic stability. It is easy to give an example to show that, when $k-d_{j}^{i}(k) \nrightarrow+\infty$, the positive system (1) may be unstable. For example, one can verify that

$$
x(k+1)=0.6 x(k-\lfloor|k \sin k|\rfloor), \quad k \in \mathbb{N}_{0},
$$

is not asymptotically stable. The main objectives of this paper are therefore to $(i)$ provide a necessary and sufficient condition for asymptotic stability of the positive system (1) under a general class of heterogeneous time-varying delays satisfying Assumption 1; and to ( $i i)$ give explicit estimates of decay rates of the positive system (1) for different classes of unbounded delays (e.g., Assumption 2).

Remark 1 Assumption 1 implies that there exists a sufficiently large $M \in \mathbb{N}$ such that $k-d_{j}^{i}(k)>0$ for all $k>M$. Let

$$
d_{\max }=-\inf _{0 \leq k \leq M}\left\{k-d_{j}^{i}(k)\right\} .
$$

Clearly, $d_{\max } \geq 0$ is bounded. It follows that, even for unbounded delays satisfying Assumption 1, it suffices to define $\varphi(\cdot)$ on a bounded set $\left\{-d_{\max }, \ldots, 0\right\}$.

\section{MAin Results}

Having established our notation and problem formulation, we will now present the main contributions of the paper.

\section{A. Asymptotic Stability of Positive Linear Systems}

The following theorem is our first key result, which states a necessary and sufficient condition for asymptotic stability of discrete-time positive linear systems with bounded or unbounded time-varying delays.

Theorem 1 For the positive system (1), suppose Assumption 1 holds. The following statements are equivalent.

(a) There exists a vector $\boldsymbol{v}>\mathbf{0}$ such that

$$
\sum_{j=1}^{n} \frac{1}{v_{i}}\left(a_{i j}+b_{i j}\right) v_{j}<1, \quad i=1, \ldots, n .
$$

(b) The positive system (1) is globally asymptotically stable for every non-negative initial condition $\varphi(\cdot)$, and for all time delays satisfying Assumption 1.

Proof: $\quad(a) \Rightarrow(b)$ : Suppose that there exists a vector $\boldsymbol{v}>\mathbf{0}$ such that (4) holds. We shall first use perfect induction to prove that for any non-negative initial condition $\varphi(\cdot) \in \mathbb{R}_{+}^{n}$, the corresponding solution $\boldsymbol{x}(k)$ of (1) satisfies $\|\boldsymbol{x}(k)\|_{\infty}^{\boldsymbol{v}} \leq\|\boldsymbol{\varphi}\|$ for all $k \geq 0$, where

$$
\|\boldsymbol{\varphi}\|=\sup _{-d_{\max } \leq s \leq 0}\|\boldsymbol{\varphi}(s)\|_{\infty}^{\boldsymbol{v}} .
$$

By the definition of $\|\varphi\|$, the initial state $\boldsymbol{x}(0)$ satisfies $\|\boldsymbol{x}(0)\|_{\infty}^{\boldsymbol{v}} \leq\|\boldsymbol{\varphi}\|$, so the induction hypothesis is true for $k=0$. Now, assume that it holds for all $k$ up to 
some $\bar{k}$, i.e., $\|\boldsymbol{x}(k)\|_{\infty}^{\boldsymbol{v}} \leq\|\boldsymbol{\varphi}\|$ for $k \leq \bar{k}$. It follows from the definition of weighted $l_{\infty}$ norm that

$$
\begin{aligned}
\frac{1}{v_{j}} x_{j}(k) & \leq\|\boldsymbol{x}(k)\|_{\infty}^{\boldsymbol{v}} \\
& \leq\|\boldsymbol{\varphi}\|, \quad j=1, \ldots, n, \quad k=-d_{\max }, \ldots, \bar{k},
\end{aligned}
$$

and hence,

$$
\begin{aligned}
\frac{1}{v_{j}} x_{j}(\bar{k}) & \leq\|\boldsymbol{\varphi}\|, \\
\frac{1}{v_{j}} x_{j}\left(\bar{k}-d_{j}^{i}(\bar{k})\right) & \leq\|\boldsymbol{\varphi}\| .
\end{aligned}
$$

For each $i \in\{1, \ldots, n\}$, we then have

$$
\begin{aligned}
\frac{1}{v_{i}} x_{i}(\bar{k}+1) & =\sum_{j=1}^{n} \frac{1}{v_{i}} a_{i j} x_{j}(\bar{k})+\sum_{j=1}^{n} \frac{1}{v_{i}} b_{i j} x_{j}\left(\bar{k}-d_{j}^{i}(\bar{k})\right) \\
& \leq\left(\sum_{j=1}^{n} \frac{1}{v_{i}} a_{i j} v_{j}+\sum_{j=1}^{n} \frac{1}{v_{i}} b_{i j} v_{j}\right)\|\varphi\| \\
& <\|\varphi\|,
\end{aligned}
$$

where we used (5) to get the first inequality, and (4) to obtain the second inequality. Therefore,

$$
\|\boldsymbol{x}(\bar{k}+1)\|_{\infty}^{\boldsymbol{v}} \leq\|\boldsymbol{\varphi}\|
$$

The induction proof is complete.

We now prove that for any non-negative initial condition $\boldsymbol{\varphi}(\cdot) \in \mathbb{R}_{+}^{n}$, the corresponding solution $\boldsymbol{x}(k)$ converges zero as $k \rightarrow \infty$. Define

$$
\gamma=\max _{i=1, \ldots, n}\left\{\sum_{j=1}^{n} \frac{1}{v_{i}}\left(a_{i j}+b_{i j}\right) v_{j}\right\} .
$$

From (4), it is clear that $\gamma \in(0,1)$. We show by induction that for each $m \in \mathbb{N}_{0}$, there exists $k_{m} \in \mathbb{N}_{0}$ such that

$$
\boldsymbol{x}(k) \in V(m), \text { for all } k \geq k_{m},
$$

where the sets $V(m)$ are defined as

$$
V(m)=\left\{\boldsymbol{x} \in \mathbb{R}^{n} \mid\|\boldsymbol{x}\|_{\infty}^{\boldsymbol{v}} \leq \gamma^{m}\|\boldsymbol{\varphi}\|\right\}, \quad m \in \mathbb{N}_{0} .
$$

According to the first part of the proof, $\|\boldsymbol{x}(k)\|_{\infty}^{\boldsymbol{v}} \leq\|\boldsymbol{\varphi}\|$ for all $k \in \mathbb{N}_{0}$, which implies that

$$
\boldsymbol{x}(k) \in V(0), \quad \forall k \geq 0 .
$$

Set $k_{0}=0$. Thus, the induction hypothesis holds for $m=0$. Assume that it is true for a given $m$, we will show that there exists $k_{m+1} \in \mathbb{N}_{0}$ such that $\boldsymbol{x}(k) \in V(m+1)$ for all $k \geq k_{m+1}$.

By Assumption $1, \lim _{k \rightarrow \infty} k-d_{j}^{i}(k)=+\infty$ for all $i, j$, so one can find a sufficiently large $\bar{m} \in \mathbb{N}$ such that

$$
k-d_{j}^{i}(k) \geq k_{m}, \quad \forall k \geq \bar{m} .
$$

Since $\boldsymbol{x}(k) \in V(m)$ for all $k \geq k_{m}$, we have

$$
\boldsymbol{x}\left(k-d_{j}^{i}(k)\right) \in V(m), \quad \forall k \geq \bar{m},
$$

which implies that

$$
\begin{aligned}
\frac{1}{v_{j}} x_{j}\left(k-d_{j}^{i}(k)\right) & \leq\left\|\boldsymbol{x}\left(k-d_{j}^{i}(k)\right)\right\|_{\infty}^{\boldsymbol{v}} \\
& \leq \gamma^{m}\|\boldsymbol{\varphi}\|, \quad \forall k \geq \bar{m} .
\end{aligned}
$$

For each $i$, we then have

$$
\begin{aligned}
\frac{1}{v_{i}} x_{i}(k+1) & \leq \sum_{j=1}^{n} \frac{1}{v_{i}} a_{i j} x_{j}(k)+\sum_{j=1}^{n} \frac{1}{v_{i}} b_{i j} x_{j}\left(k-d_{j}^{i}(k)\right) \\
& \leq\left(\sum_{j=1}^{n} \frac{1}{v_{i}} a_{i j} v_{j}+\sum_{j=1}^{n} \frac{1}{v_{i}} b_{i j} v_{j}\right) \gamma^{m}\|\boldsymbol{\varphi}\| \\
& \leq \gamma^{m+1}\|\varphi\|, \quad \forall k \geq \bar{m},
\end{aligned}
$$

where we used (7) to get the second inequality, and (6) to obtain the last inequality. Therefore,

$$
\|\boldsymbol{x}(k+1)\|_{\infty}^{\boldsymbol{v}} \leq \gamma^{m+1}\|\boldsymbol{\varphi}\|, \quad \forall k \geq \bar{m}
$$

which implies that $\boldsymbol{x}(k+1) \in V(m+1)$ for all $k \geq \bar{m}$. Thus,

$$
\boldsymbol{x}(k) \in V(m+1), \quad \forall k \geq \bar{m}+1 .
$$

Set $k_{m+1}=\bar{m}+1$. This completes the induction proof.

In summary, we conclude that for each $m$, there exists $k_{m}$ such that $\boldsymbol{x}(k) \in V(m)$ for all $k \geq k_{m}$. Since $\gamma<1, \gamma^{m}$ approaches zero as $m \rightarrow+\infty$, and hence $\boldsymbol{x}(k)$ converges zero as $k \rightarrow \infty$.

$(b) \Rightarrow(a)$ : Suppose (1) is globally asymptotically stable for all delays satisfying Assumption 1. Particularly, let $d_{j}^{i}(k)=d$ for $i, j=1, \ldots, n$, where $d<\infty$ is a positive constant. Then, the positive linear system

$$
\boldsymbol{x}(k+1)=A \boldsymbol{x}(k)+B \boldsymbol{x}(k-d), \quad k \in \mathbb{N}_{0},
$$

is asymptotically stable. The conclusion follows from [12, Theorem 2].

According to Theorem 1, the positive system (1) is asymptotically stable for all delays satisfying Assumption 1 if and only if the following set of linear inequalities in $\boldsymbol{v}$,

$$
\left\{\begin{array}{cc}
(A+B) \boldsymbol{v} & <\boldsymbol{v} \\
\boldsymbol{v} & >\mathbf{0}
\end{array}\right.
$$

is feasible. The above stability condition is a linear programming (LP) problem in $\boldsymbol{v}$, and thus can be verified numerically in polynomial time. Since $A$ and $B$ are non-negative, $A+B$ is also non-negative. According to property of non-negative matrices [5, Proposition 1], inequality (8) holds if and only if $\rho(A+B)<1$. This means that if the positive system

$$
\boldsymbol{x}(k+1)=(A+B) \boldsymbol{x}(k)
$$

has an asymptotically stable equilibrium at the origin, it remains asymptotically stable under any heterogeneous timevarying delays satisfying Assumption 1.

Remark 2 Previous works in the literature established necessary and sufficient conditions for asymptotic stability of discrete-time positive linear systems, but required that the 
delays be either bounded (e.g., [11]-[13]), or unbounded under Assumption 2 [14]. It is clear that Assumption 1 covers all these classes of time-delays as special cases. Hence, we prove the asymptotic stability of positive linear systems under much less conservative assumption.

\section{B. Polynomial-Rate Stability of Positive Linear Systems}

Theorem 1 is concerned with the asymptotic stability of positive linear systems with delays. However, there are processes and applications for which it is desirable that the system has a certain decay rate. Loosely speaking, the system has to converge quickly enough to the equilibrium. Hence, it is important to investigate the impact of delays on the decay rate of such systems. While the asymptotic stability of the positive system (1) with unbounded delays satisfying Assumption 2 has been investigated in [14], the impact of delays on the decay rate has been missing. In this section, we therefore determine how unbounded delays affect the decay rate of positive linear systems. Before stating the main result, we provide the definition of polynomial-rate stability for the discrete-time systems.

Definition 1 The discrete-time system (1) is said to be globally polynomial-rate stable if there exists a scalar $\xi>0$, such that for any initial condition $\varphi(\cdot)$, the solution $\boldsymbol{x}(k)$ of (1) satisfies $\|\boldsymbol{x}(k)\|=O\left(k^{-\xi}\right)$ for all $k \in \mathbb{N}$, which means that there exist scalars $M>0$ and $\xi>0$ such that

$$
\|\boldsymbol{x}(k)\| \leq M k^{-\xi}, \quad \forall k \in \mathbb{N} .
$$

The following theorem shows that under Assumption 2, the positive system (1) is globally polynomial-rate stable.

Theorem 2 For the positive system (1), suppose Assumption 2 holds. If there exists a vector $\boldsymbol{v}>\mathbf{0}$ satisfying

$$
\sum_{j=1}^{n} \frac{1}{v_{i}}\left(a_{i j}+b_{i j}\right) v_{j}<1,
$$

for all $i$, then the positive system (1) is globally polynomialrate stable. In particular,

$$
\|\boldsymbol{x}(k)\|_{\infty}^{\boldsymbol{v}}=O\left(k^{-\xi}\right), \quad k \in \mathbb{N},
$$

where $\xi_{i}$ is the unique positive solution of the equation

$$
\left(\sum_{j=1}^{n} \frac{1}{v_{i}} a_{i j} v_{j}\right)+\left(\sum_{j=1}^{n} \frac{1}{v_{i}} b_{i j} v_{j}\right)\left(\frac{1}{1-\alpha}\right)^{\xi_{i}}=1,
$$

and $0<\xi<\min _{1 \leq i \leq n} \xi_{i}$.

Before the proof of Theorem 2 is presented, it is worth making a remark regarding Equation (11).

Remark 3 Equation (11) has three parameters, namely, the positive vector $\boldsymbol{v}, \alpha$, and $\xi_{i}$. For any fixed $0<\alpha<1$, and any fixed $\boldsymbol{v}>\mathbf{0}$, (11) is a nonlinear equation with respect to $\xi_{i}$. The left-hand side of (11) is strictly monotonically increasing in $\xi_{i}>0$ and, by (9), is smaller than the righthand side for $\xi_{i}=0$. Therefore, (11) always admits a unique positive solution $\xi_{i}$.
Proof of Theorem 3: According to Remark 3, Equation (11) has a unique positive solution $\xi_{i}$. Pick a constant $\xi>0$ satisfying $0<\xi<\min _{1 \leq i \leq n} \xi_{i}$. Since the left-hand side of (11) is monotonically increasing in $\xi_{i}$, we have

$$
\left(\sum_{j=1}^{n} \frac{1}{v_{i}} a_{i j} v_{j}\right)+\left(\sum_{j=1}^{n} \frac{1}{v_{i}} b_{i j} v_{j}\right)\left(\frac{1}{1-\alpha}\right)^{\xi}<1,
$$

for all $i$. It follows that

$$
\begin{aligned}
\left(\sum_{j=1}^{n} \frac{1}{v_{i}} a_{i j} v_{j}\right) & \left(\lim _{k \rightarrow \infty} \frac{k+1}{k}\right)^{\xi} \\
& +\left(\sum_{j=1}^{n} \frac{1}{v_{i}} b_{i j} v_{j}\right)\left(\lim _{k \rightarrow \infty} \frac{k+1}{(1-\alpha) k}\right)^{\xi}<1 .
\end{aligned}
$$

Thus, there exists a sufficiently large number $T^{\prime} \in \mathbb{N}$ such that

$$
\begin{aligned}
& \left(\sum_{j=1}^{n} \frac{1}{v_{i}} a_{i j} v_{j}\right)\left(\frac{1}{k}\right)^{\xi} \\
& \quad+\left(\sum_{j=1}^{n} \frac{1}{v_{i}} b_{i j} v_{j}\right)\left(\frac{1}{(1-\alpha) k}\right)^{\xi} \leq\left(\frac{1}{k+1}\right)^{\xi}
\end{aligned}
$$

holds for all $k>T^{\prime}$. On the other hand, from Assumption 2, there exists a number $T \in \mathbb{N}$ such that

$$
\frac{1}{k-d_{j}^{i}(k)} \leq \frac{1}{(1-\alpha) k}, \quad \forall i, j, \forall k>T,
$$

which implies that

$$
\left(\frac{1}{k-d_{j}^{i}(k)}\right)^{\xi} \leq\left(\frac{1}{(1-\alpha) k}\right)^{\xi}, \quad \forall k>T .
$$

Let $\bar{T}=\max \left\{T, T^{\prime}\right\}+1$. Combining (12) and (13) yields

$$
\begin{aligned}
& \left(\sum_{j=1}^{n} \frac{1}{v_{i}} a_{i j} v_{j}\right)\left(\frac{1}{k}\right)^{\xi} \\
& +\sum_{j=1}^{n}\left(\left(\frac{1}{v_{i}} b_{i j} v_{j}\right)\left(\frac{1}{k-d_{j}^{i}(k)}\right)^{\xi}\right) \leq\left(\frac{1}{k+1}\right)^{\xi}
\end{aligned}
$$

for all $k \geq \bar{T}$. Define

$$
M=\max _{k=1, \ldots, \bar{T}}\left\{k^{\xi}\|\boldsymbol{x}(k)\|_{\infty}^{\boldsymbol{v}}\right\} .
$$

We now use induction to prove that $\|\boldsymbol{x}(k)\|_{\infty}^{\boldsymbol{v}} \leq M k^{-\xi}$ for all $k \in \mathbb{N}$. From the definition of $M$, it is clear that the induction hypothesis is true for $k=1, \ldots, \bar{T}$. Next, assume that it holds for all $k$ up to some $m$, where $m>\bar{T}$. Thus

$$
\|\boldsymbol{x}(k)\|_{\infty}^{\boldsymbol{v}} \leq M k^{-\xi}, \quad k=1, \ldots, m,
$$

which implies that

$$
\begin{aligned}
\frac{1}{v_{j}} x_{j}(m) & \leq \frac{M}{m^{\xi}}, \\
\frac{1}{v_{j}} x_{j}\left(m-d_{j}^{i}(m)\right) & \leq \frac{M}{\left(m-d_{j}^{i}(m)\right)^{\xi}},
\end{aligned}
$$


for $j=1, \ldots, n$. We will show that $\boldsymbol{x}(m+1)$ satisfies (10). For each $i$, we have

$$
\begin{aligned}
\frac{1}{v_{i}} x_{i}(m+1) & =\sum_{j=1}^{n} \frac{1}{v_{i}} a_{i j} x_{j}(m)+\sum_{j=1}^{n} \frac{1}{v_{i}} b_{i j} x_{j}\left(m-d_{j}^{i}(m)\right) \\
\leq & \left(\sum_{j=1}^{n} \frac{1}{v_{i}} a_{i j} v_{j}\right)\left(\frac{M}{m^{\xi}}\right) \\
& \quad+\sum_{j=1}^{n}\left(\left(\frac{1}{v_{i}} b_{i j} v_{j}\right)\left(\frac{M}{\left(m-d_{j}^{i}(m)\right)^{\xi}}\right)\right) \\
\leq & \frac{M}{(m+1)^{\xi}},
\end{aligned}
$$

where we used (16) to get the first inequality, and (14) to obtain the second inequality. Therefore,

$$
\|\boldsymbol{x}(m+1)\|_{\infty}^{\boldsymbol{v}} \leq \frac{M}{(m+1)^{\xi}} .
$$

This completes the proof.

In Equation (11), $\xi_{i}$ is monotonically decreasing with $\alpha$ and approaches zero as $\alpha$ tends to one. Hence, while the positive system (1) remains polynomial-rate stable for arbitrary unbounded delays satisfying Assumption 2, the decay rate deteriorates with increasing $\alpha$. In other words, the decay rate gets slower if delays grow more quickly as $k \rightarrow \infty$.

As shown in Theorem 2, the positive system (1) with unbounded delays satisfying Assumption 2 is polynomialrate stable if the LP problem (8) is feasible. Moreover, any vector $\boldsymbol{v}$ that satisfies the inequalities (8) can be used to find a guaranteed rate of convergence of (1) by computing the associated $\xi$ in (11). It is easily seen that the guaranteed decay rate depends on the choice of vector $\boldsymbol{v}$. Next, we will show that the best decay rate that our results can ensure, along with the associated vector $\boldsymbol{v}$ can be found via convex optimization. To this end, we use the logarithmic change of variables $z_{i}=\ln \left(v_{i}\right)$ for $i=1, \ldots, n$. Then, the search for $\boldsymbol{v}$ can be formulated as

\section{maximize $\quad \xi$ subject to:}

$$
\begin{aligned}
& \xi<\xi_{i}, \\
& \sum_{j=1}^{n}\left(a_{i j}+b_{i j}\right) e^{z_{j}-z_{i}}<1 \\
& \sum_{j=1}^{n} a_{i j} e^{z_{j}-z_{i}}+\sum_{j=1}^{n} b_{i j} e^{z_{j}-z_{i}+\left(\ln \frac{1}{1-\alpha}\right) \xi_{i}} \leq 1
\end{aligned}
$$

$$
i=1, \ldots, n
$$

where the last two constraints are (9) and (11) in the new variables, respectively. The optimization variables are the vector $\boldsymbol{z}=\left[z_{1}, \ldots, z_{n}\right]^{T}$ and the decay rate $\xi$. Since $a_{i j}, b_{i j} \geq 0$ for all $i, j$, the last two constraints in (17) are convex in $\xi$ and $z$. This implies that this is a convex optimization problem, hence it can be efficiently solved.

\section{Beyond Polynomial-Rate Stability}

While the stability of positive linear systems with unbounded delays satisfying Assumption 1 may, in general, only be asymptotic, Theorem 2 demonstrates that if the delays grow sufficiently slowly, we can guarantee a certain decay rate. However, the linear bound $d_{j}^{i}(k) \leq \alpha k$ on the delays used in Assumption 2 is rather arbitrary. To make our point, we will establish convergence rates for another class of unbounded delays that do not satisfy Assumption 2. These delays are described by the following assumption.

Assumption 3 There exist $T \in \mathbb{N}$ and a scalar $0<\beta<1$ such that

$$
d_{j}^{i}(k) \leq k-\left(\frac{k}{\ln (k)}\right)^{1-\beta}, \quad \forall k>T, i, j=1, \ldots, n .
$$

Theorem 3 Consider the positive system (1) under Assumption 3. Suppose that there exists a vector $\boldsymbol{v}>\mathbf{0}$ satisfying (9). Let $\lambda_{i}$ be the unique positive solution of the equation

$$
\left(\sum_{j=1}^{n} \frac{1}{v_{i}} a_{i j} v_{j}\right)+\left(\sum_{j=1}^{n} \frac{1}{v_{i}} b_{i j} v_{j}\right)\left(\frac{1}{1-\beta}\right)^{\lambda_{i}}=1 .
$$

Then, every solution $\boldsymbol{x}(k)$ of (1) satisfies the condition

$$
\|\boldsymbol{x}(k)\|_{\infty}^{\boldsymbol{v}} \leq \frac{M}{(\ln (k+1))^{\lambda}}, \quad k \in \mathbb{N},
$$

where $M$ is a positive constant, and $0<\lambda<\min _{1 \leq i \leq n} \lambda_{i}$.

Proof: For any fixed $0<\beta<1$, and any fixed $\boldsymbol{v}>\mathbf{0}$, Equation (18) is a nonlinear equation with respect to $\lambda_{i}$. According to Remark 3, (18) has a unique positive solution $\lambda_{i}$. Therefore, one can find a constant $\lambda>0$ satisfying $0<\lambda<\min _{1 \leq i \leq n} \lambda_{i}$. The left-hand side of (18) is monotonically increasing in $\lambda_{i}$ which implies that

$$
\left(\sum_{j=1}^{n} \frac{1}{v_{i}} a_{i j} v_{j}\right)+\left(\sum_{j=1}^{n} \frac{1}{v_{i}} b_{i j} v_{j}\right)\left(\frac{1}{1-\beta}\right)^{\lambda}<1 .
$$

Since $\lim _{k \rightarrow \infty} \frac{\ln (k+1)}{\ln \frac{k}{\ln k}}=1$, it follows from (19) that

$$
\begin{aligned}
\left(\sum_{j=1}^{n} \frac{1}{v_{i}} a_{i j} v_{j}\right) & \left(\lim _{k \rightarrow \infty} \frac{\ln (k+1)}{\ln (k)}\right)^{\lambda} \\
+ & \left(\sum_{j=1}^{n} \frac{1}{v_{i}} b_{i j} v_{j}\right)\left(\lim _{k \rightarrow \infty} \frac{\ln (k+1)}{(1-\beta) \ln \frac{k}{\ln k}}\right)^{\lambda}<1 .
\end{aligned}
$$

Therefore, there exists a sufficiently large number $T^{\prime} \in \mathbb{N}$, such that for all $k>T^{\prime}$, it holds

$$
\begin{aligned}
& \left(\sum_{j=1}^{n} \frac{1}{v_{i}} a_{i j} v_{j}\right)\left(\frac{1}{\ln k}\right)^{\lambda} \\
& \quad+\left(\sum_{j=1}^{n} \frac{1}{v_{i}} b_{i j} v_{j}\right)\left(\frac{1}{(1-\beta) \ln \frac{k}{\ln k}}\right)^{\lambda} \leq\left(\frac{1}{\ln (k+1)}\right)^{\lambda} .
\end{aligned}
$$

By Assumption 3, there exists a number $T \in \mathbb{N}$ such that

$$
\left(\frac{k}{\ln (k)}\right)^{1-\beta} \leq k-d_{j}^{i}(k), \quad \forall i, j, \forall k>T,
$$


which implies that

$$
\frac{1}{\ln \left(k-d_{j}^{i}(k)\right)} \leq \frac{1}{(1-\beta) \ln \frac{k}{\ln k}}, \quad \forall k>T .
$$

Let $\bar{T}=\max \left\{T, T^{\prime}\right\}+1$. The rest of the proof is similar to the proof of Theorem 2 and thus we omit it.

Theorem 3 shows that the decay rate of the positive system (1) with unbounded delays satisfying Assumption 3 is of the order $O\left((\ln (k+1))^{-\lambda}\right)$, and any feasible solution $\boldsymbol{v}$ to the LP problem (8) can be used to find a guaranteed decay rate by computing the associated $\lambda$ in (18). In order to find the best decay rate that our bound can provide, we can again solve the convex optimization problem (17). The only change is that we replace $\xi$ with $\lambda$, and $\alpha$ with $\beta$.

According to theorems $1-3$, we see that even though the delays are very large, the stability condition (8) can still be necessary and sufficient for asymptotic stability of the positive system (1). On the other hand, the quicker the delays are allowed to tend to infinity, the longer the guaranteed convergence times. Specifically, the positive system (1) is polynomial-rate stable if Assumption 2 holds, and log-rate stable if the delays satisfy Assumption 3.

\section{An Illustrative Example}

Consider the system described by (1) with

$$
A=\left[\begin{array}{ll}
0.20 & 0.15 \\
0.10 & 0.20
\end{array}\right], B=\left[\begin{array}{ll}
0.15 & 0.10 \\
0.10 & 0.20
\end{array}\right]
$$

Since $A$ and $B$ are non-negative, by Proposition 1 , the system (20) is positive. Moreover, it is easy to verify that $\rho(A+B)<1$. Therefore, according to Theorem 1, (20) is globally asymptotically stable for any delays satisfying Assumption 1. For example, we take

$$
d_{j}^{i}(k)=k-\left\lfloor\frac{k}{\ln (k+2)}\right\rfloor, k \in \mathbb{N}_{0}, i, j=1,2,
$$

and the simulation result is shown in Figure 1, from which one can see that the positive system (20) is indeed asymptotically stable. Note that Theorem 1 in [14] can not be applied in this example, since the delays $d_{j}^{i}(k)$ do not satisfy Assumption 2.

\section{Conclusions}

In this paper, we addressed the delay-independent stability of discrete-time positive linear systems with heterogeneous unbounded time-varying delays and derived a necessary and sufficient condition for asymptotic stability. For two particular classes of unbounded delays, explicit expressions that bound the decay rate of positive linear systems were presented. We demonstrated that the best decay rate that our bounds can provide can be easily found via convex optimization techniques. Finally, the validity of the results was demonstrated via a simple example.

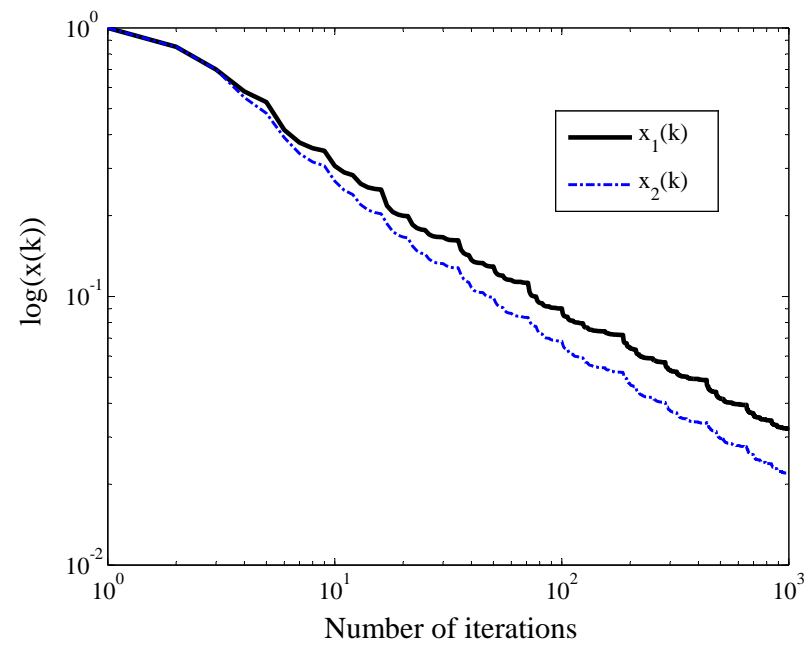

Fig. 1. Illustration of the asymptotic stability for the positive linear system (20). The horizontal axis represents the number of iterations and the vertical axis denotes logarithm of the state variable.

\section{REFERENCES}

[1] L. Farina and S. Rinaldi, Positive Linear Systems: Theory and Applications. John Wiley and Sons, New York, 2000.

[2] P. D. Leenheer and D. Aeyels, "Stabilization of positive linear systems," Systems and Control Letters, vol. 44, pp. 259-271, 2001.

[3] M. A. Rami and F. Tadeo, "Controller synthesis for positive linear systems with bounded controls," IEEE Transactions on Circuits and Systems II, vol. 54, no. 2, pp. 151-155, Feb. 2007.

[4] W. M. Haddad, V. Chellaboina, and Q. Hui, Nonnegative and Compartmental Dynamical Systems. Princeton, New Jersey, 2010.

[5] A. Rantzer, "Distributed control of positive systems," 50th IEEE Conference on Decision and Control and European Control Conference (CDC-ECC), pp. 6608-6611, 2011.

[6] C. Briat, "Robust stability analysis of uncertain linear positive systems via integral linear constraints: $L_{1}$ - and $L_{\infty}$-gain characterizations," 50th IEEE Conference on Decision and Control and European Control Conference (CDC-ECC), pp. 6337-6342, 2011.

[7] T. Tanaka and C. Langbort, "The bounded real lemma for internally positive systems and H-Infinity structured static state feedback," IEEE Transactions on Automatic Control, vol. 56, no. 9, pp. 2218-2223, 2011.

[8] E. Fornasini and M. E. Valcher, "Stability and stabilizability criteria for discrete-time positive switched systems," IEEE Transactions on Automatic Control, vol. 57, no. 5, pp. 1208-1221, 2012.

[9] H. R. Feyzmahdavian, T. Charalambous, and M. Johansson, "On the rate of convergence of continuous-time linear positive systems with heterogeneous time-varying delays," European Control Conference (ECC), pp. 3372-3377, July 2013.

[10] R. D. Driver, Ordinary and Delay Differential Equations. Springer, New York, 1977.

[11] W. Haddad and V. Chellaboina, "Stability theory for non-negative and compartmental dynamical systems with time delay," Syst. Control Lett., vol. 51, no. 5, pp. 355-361, 2004.

[12] M. Buslowicz, "Simple stability conditions for linear positive discretetime systems with delays," Bulletin of the Polish Academy of Sciences: Technical Sciences, vol. 56, pp. 325-328, 2008.

[13] X. Liu, W. Yu, and L. Wang, "Stability analysis of positive systems with bounded time-varying delays," IEEE Transactions on Circuits and Systems II, vol. 56, no. 7, pp. 600-604, July 2009.

[14] X. Liu and C. Dang, "Stability analysis of positive switched linear systems with delays," Automatic Control, IEEE Transactions on, vol. 56, no. 7, pp. 1684-1690, July 2011. 